\title{
Diabetes mellitus tipo 2 versus potencial antioxidante do pequi: uma revisão
}

\author{
Type 2 Diabetes mellitus versus pequil's antioxidant potential: a review \\ Diabetes mellitus tipo 2 versus potencial antioxidante de pequi: una revisión
}

Recebido: 19/08/2021 | Revisado: 25/08/2021 | Aceito: 30/08/2021 | Publicado: 02/09/2021

\author{
Laís Lima de Castro Abreu \\ ORCID: https://orcid.org/0000-0001-6909-6774 \\ Universidade Federal do Piauí, Brasil \\ E-mail: lais.castro123@ufpi.edu.br \\ Rodrigo Feijão Rolim \\ ORCID: https://orcid.org/0000-0003-4863-9295 \\ Universidade Federal do Piauí, Brasil \\ E-mail: rodrigofeijjao2016@ hotmail.com \\ Alisson de Abreu Almeida \\ ORCID: https://orcid.org/0000-0003-1219-2305 \\ Empresa Social de Regularização Fundiária, Piauí \\ E-mail: alissonabreu.adv@gmail.com
}

\begin{abstract}
Resumo
O diabetes mellitus tipo 2 (DM2) caracteriza-se pela intolerância à glicose e hiperglicemia, o que favorece a manifestação do estresse oxidativo e inflamação, condições consideradas como um dos principais fatores envolvidos na fisiopatologia das complicações diabéticas. Medidas que possam reduzir o estresse oxidativo e a inflamação constituem-se em uma estratégia viável para o controle do DM2. O artigo teve como objetivo identificar a relação entre o potencial antioxidante do pequi (Caryocar brasiliense Camb) e a prevenção do processo inflamatório/oxidativo presente no DM2. Trata-se de uma revisão de literatura de caráter narrativo, na qual realizou-se uma pesquisa bibliográfica durantes os meses de janeiro a junho de 2021 nas bases de dados PubMed, Scielo e portal periódicos CAPES, utilizando os termos descritos no DeSC: Pequi, Diabetes Mellitus tipo 2, Inflamação e Estresse oxidativo, em português, inglês e espanhol. A partir da leitura, observou-se que o pequi é um fruto rico em ácidos graxos, tais como os ácidos oleico, palmítico e esteárico, sendo que pesquisas recentes indicam que esse fruto também possui quantidades significativas de carotenoides e compostos fenólicos, demonstrando um forte potencial antioxidante natural, com propriedades comerciais, e possíveis aplicações tecnológicas e funcionais exploráveis. O consumo de alimentos fontes de antioxidantes como frutos do cerrado, especificamente o pequi podem contribuir para prevenção da cascata patológica presente no DM2, entretanto são necessários mais estudos que verifiquem a influência dos compostos antioxidantes presentes no óleo de pequi sobre o sistema de defesa antioxidante e atividade inflamatória na presença de hiperglicemia crônica.
\end{abstract}

Palavras-chave: Diabetes mellitus tipo 2; Estresse oxidativo; Inflamação; Pequi.

\begin{abstract}
Type 2 diabetes mellitus (DM2) is characterized by glucose intolerance and hyperglycemia, which favors the manifestation of oxidative stress and inflammation, conditions considered as one of the main factors involved in the pathophysiology of diabetic complications. Measures that can reduce oxidative stress and inflammation constitute a viable strategy for the control of DM2. The article aimed to identify the relationship between the antioxidant potential of pequi (Caryocar brasiliense Camb) and the prevention of the inflammatory/oxidative process present in DM2. This is a literature review of a narrative nature, in which a literature search was carried out from January to June 2021 in the PubMed, Scielo and CAPES journals databases, using the terms described in DeSC: Pequi, Type 2 Diabetes Mellitus, Inflammation and Oxidative Stress, in Portuguese, English and Spanish. From the reading, it was observed that pequi is a fruit rich in fatty acids, such as oleic, palmitic and stearic acids, and recent research indicates that this fruit also has significant amounts of carotenoids and phenolic compounds, demonstrating a strong natural antioxidant potential, with commercial properties, and possible exploitable technological and functional applications. The consumption of foods that are sources of antioxidants such as cerrado fruits, specifically pequi, can contribute to the prevention of the pathological cascade present in DM2, however more studies are needed to verify the influence of antioxidant compounds present in pequi oil on the antioxidant defense system and inflammatory activity in the presence of chronic hyperglycemia.
\end{abstract}

Keywords: Type 2 Diabetes mellitus; Oxidative stress; Inflammation; Pequi. 


\begin{abstract}
Resumen
La diabetes mellitus tipo 2 (DM2) se caracteriza por la intolerancia a la glucosa y la hiperglucemia, lo que favorece la manifestación del estrés oxidativo y la inflamación, condiciones consideradas como uno de los principales factores implicados en la fisiopatología de las complicaciones diabéticas. Las medidas que pueden reducir el estrés oxidativo y la inflamación constituyen una estrategia viable para el control de la DM2. El artículo tuvo como objetivo identificar la relación entre el potencial antioxidante del pequi (Caryocar brasiliense Camb) y la prevención del proceso inflamatorio / oxidativo presente en la DM2. Se trata de una revisión bibliográfica de carácter narrativo, en la que se realizó una búsqueda bibliográfica de enero a junio de 2021 en las bases de datos de las revistas PubMed, Scielo y CAPES, utilizando los términos descritos en DeSC: Pequi, Diabetes Mellitus Tipo 2, Inflamación y Oxidativa. Stress, en portugués, inglés y español. A partir de la lectura, se observó que el pequi es una fruta rica en ácidos grasos, como los ácidos oleico, palmítico y esteárico, e investigaciones recientes indican que esta fruta también tiene cantidades importantes de carotenoides y compuestos fenólicos, demostrando un fuerte potencial antioxidante natural. con propiedades comerciales, y posibles aplicaciones tecnológicas y funcionales explotables. El consumo de alimentos fuente de antioxidantes como los frutos del cerrado, específicamente el pequi, puede contribuir a la prevención de la cascada patológica presente en la DM2, sin embargo se necesitan más estudios para verificar la influencia de los compuestos antioxidantes presentes en el aceite de pequi en la defensa antioxidante sistema y actividad inflamatoria en presencia de hiperglucemia crónica.
\end{abstract}

Palabras clave: Diabetes mellitus tipo 2; Estrés oxidativo; Inflamación; Pequi.

\title{
1. Introdução
}

Ao longo dos dois últimos séculos, a revolução tecnológica e industrial trouxe consequências econômicas e sociais que resultaram em uma mudança drástica do perfil de morbimortalidade da população, com grande predomínio das doenças e mortes devidas às doenças crônicas não transmissíveis (DCNT), dentre elas o câncer, diabetes mellitus e as doenças cardiovasculares (Ministério da Saúde, 2006). Em consequência do aumento dessas doenças, observa-se um elevado custo com tratamentos de saúde e previdência social, além de graves repercussões para as famílias e as pessoas portadoras de DCNT.

O diabetes mellitus (DM) é considerado como um grupo de doenças metabólicas resultante de um estado de hiperglicemia crônica decorrente de defeitos na secreção e/ou ação da insulina (American Diabetes Association, 2014). Ao longo do tempo, essa condição de hiperglicemia provoca complicações microvasculares (por exemplo, retinopatia, neuropatia e nefropatia) e macrovasculares (infarto do miocárdio, doença vascular periférica, e acidente vascular cerebral), sendo estas consideradas como a principal causa de morte nos diabéticos (Johnson, 2012; Paneni et al., 2013).

Dentre os tipos de DM, o diabetes mellitus tipo 2 (DM2) é a forma mais frequente, representando 90\% a 95\% do total de casos. Esse tipo de DM caracteriza- se pela intolerância à glicose e hiperglicemia, em que a principal alteração fisiopatológica é a resistência periférica à ação da insulina associada com uma deficiência na secreção deste hormônio (Sociedade Brasileira de Diabetes, 2015).

A projeção da Internacional Diabetes Federation (IDF) para 2030 é de 552 milhões de indivíduos com DM, o que corresponderá a 9,9\% da população mundial adulta naquele período. A estimativa da Organização Mundial da Saúde (OMS) para o Brasil, para o mesmo período, é de 11,3 milhões de pessoas com diabetes, o que tornaria o país o oitavo no mundo com o maior número de pessoas portadoras da doença (Winkelmann \& Fontela, 2014).

O estado de hiperglicemia crônica favorece a manifestação do estresse oxidativo, condição considerada como um dos principais fatores envolvidos na fisiopatologia das complicações diabéticas. Esse fato pode estar relacionado com o aumento da produção de espécies reativas de oxigênio (EROS), e com a redução da atividade do sistema de defesa antioxidante do organismo (Pitocco et al., 2013; Rani \& Mythili, 2014).

Outro fator que contribui para o surgimento de complicações diabéticas, é o estado de inflamação crônica, que está implicado na patogênese do DM2 e no agravamento do quadro clínico, com aparecimento, por exemplo, de doenças cardiovasculares e retinopatia diabética (Williams et al., 2007). As respostas inflamatórias podem ter papel duplo no DM2, uma vez que levam à resistência à insulina; além de também contribuírem para intensificar o estado hiperglicêmico, resultando em complicações do DM2 (Cruz et al., 2013). 
Uma variedade de estímulos como, liberação de citocinas, hiperglicemia e hiperlipidemia, podem perturbar a homeostase, resultando em estresse oxidativo e inflamação. Estudos mostram que mecanismos inflamatórios e a produção de citocinas ativadas pelo estresse por meio da inflamação podem alterar ainda mais a estrutura das células $\beta$ pancreáticas (Montane, Cadavez \& Novials, 2014).

Nos últimos anos, evidências têm apontado que o estresse oxidativo desempenha um papel crucial no desenvolvimento e perpetuação da inflamação, sendo que essas condições se reforçam entre si, estabelecendo um ciclo vicioso capaz de perpetuar e propagar a resposta inflamatória, contribuindo na patogênese do DM e de várias outras doenças (Lugrin et al., 2014). Medidas que possam reduzir o estresse oxidativo e a inflamação constituem-se em uma estratégia viável para o controle do DM2.

A ingestão de micronutrientes com ação antioxidante tem sido associada com estado reduzido do estresse oxidativo, inflamação, e redução de risco de DM2 em diversos estudos transversais e de intervenção (Badawi et al., 2010; Garcia-Bailo et al., 2011). Estudos epidemiológicos mostram que a dieta com antioxidantes protege as células do estresse oxidativo e pode reduzir o risco de complicações do DM2 (Rafighi et al., 2013; Wedick et al., 2012).

No Brasil, há abundante oferta de oleaginosas usadas na produção de óleos vegetais; no entanto, é ainda necessário maior aproveitamento de óleos de sementes alternativas provenientes de cada região brasileira. O pequi (Caryocar brasiliense Camb), juntamente com outras novas alternativas, como pinhão manso (Jatropha curcas), nabo (Raphanus sativus L.), buriti (Mauritia flexuosa), macaúba (Acrocomia aculeata) e grande variedade de oleaginosas, surgem como espécies ainda a serem exploradas (Oliveira \& Ramalho, 2006). Dados sobre composição e propriedades físicoquímicas, além de capacidade antioxidante, para a maioria desses óleos vegetais alternativos ainda não foram relatados na literatura científica (Ramadan et al., 2012).

O pequi é encontrado no cerrado brasileiro e pertence à família Caricaceae, gênero Caryocar (Bernardes et al., 2008; Vera et al., 2007). O fruto do pequi possui uma camada de polpa amarelada que circunda uma fina camada de espinhos e, mais internamente, uma amêndoa branca que é rica em óleo (De Oliveira Sousa et al., 2011). A amêndoa é considerada um subproduto comercial inexplorado, proveniente do processamento da polpa; no entanto, ela pode ser torrada para a produção de óleo, consumida fresca ou usada como um ingrediente culinário em bolos, paçocas e pamonhas (Da Silva Rabêlo et al., 2008; Segall et al., 2006). Porém, sua frutificação ocorre apenas de novembro a fevereiro, o que limita a comercialização e o acesso da população ao pequi (Alves et al., 2008). Na medicina popular, o óleo de pequi é considerado um tônico e pode ser usado no tratamento de bronquite, resfriados, influenza e em manifestações de deficiência de vitamina A (Ribeiro et al., 2012).

A composição química da polpa de seu fruto varia conforme o clima e a região onde o pequi é plantado. Assim, há 20 a 27\% de lipídeos, 2,2 a $6 \%$ de proteínas, $11,65 \%$ de fibras e $19,7 \%$ de carboidratos, além de conter diferentes antioxidantes como carotenoides, inclusive $\beta$-caroteno, licopeno, criptoflavona, anteroxantina, zeaxantina, mutatoxantina, violoxantina, luteína e neoxantina, vitamina $\mathrm{C}$, componentes fenólicos (flavonóides e taninos). A polpa do fruto em sua composição de ácidos graxos (AG), há ácido oléico monoinsaturado (48,7 a 54\%), ácido palmítico saturado (39 a 44,3\%) além de outros constituintes em menor quantidade, como ácidos palmitoleico $(0,67 \%)$, linoléico $(1,36 \%)$, linolênico $(0,51 \%)$, esteárico $(1,28 \%)$ e araquidônico - 0,12\% (Miranda-Vilela et al., 2009). Devido ao seu baixo teor de ácidos graxos polinsaturados e alto teor de ácidos graxos monoinsaturados, o óleo de pequi pode ser usado em processos que envolvam cocção e fritura (Ribeiro et al., 2012).

O óleo da amêndoa de pequi é constituído principalmente por ácidos graxos (AG), tais como os ácidos oleico (50,2 $\%$ ), palmítico (42,3\%), e esteárico (1,5\%), e menores quantidades, o mirístico, o palmitoleico, o linoleico e o linolênico (Mata et al., 2009). Essa composição em AG assegura importante valor nutricional ao óleo da amêndoa de pequi (OAP), além de o 
consumo de ácido oleico estar relacionado com decréscimo nos níveis de lipoproteína de baixa densidade (LDL) e redução do risco de doença coronariana (Ramadan et al., 2012).

Apesar da pouca caracterização em relação aos compostos minoritários, o OAP é considerado uma boa fonte de matéria-prima para a indústria cosmética e alimentar, devido à sua composição em AG. Na medicina popular, OAP é utilizado como agente anti-inflamatório, para curar feridas e para o tratamento de dores reumáticas e musculares (De Oliveira et al., 2010; Passos et al., 2003). Pesquisas apontam para o papel do óleo na inflamação e no reparo tecidual de lesões induzidas experimentalmente (Leite et al., 2009; Quirino et al., 2009).

Sabe-se que o consumo de dietas inadequadas, ricas em gorduras saturadas, açúcar simples e sal, e pobres em frutas e verduras são atualmente o padrão alimentar de grande parcela da população; e isso repercute no estado de saúde e doença da população. Assim, é relevante a discussão a respeito de intervenções dietéticas baseadas, por exemplo, no consumo de compostos bioativos, como carotenoides, com seu potencial antioxidante, para que o conhecimento de sua ação no processo inflamatório e sobre o estresse oxidativo possa contribuir para a diminuição de suas complicações metabólicas. Dessa forma o presente artigo teve como objetivo identificar a relação entre o potencial antioxidante do pequi (Caryocar brasiliense Camb) e a prevenção do processo inflamatório/oxidativo presente no diabetes mellitus tipo 2.

\section{Metodologia}

Trata-se de um estudo qualitativo de revisão narrativa realizado durantes os meses de janeiro a junho de 2021 nas bases de dados PubMed, Scielo e portal periódicos CAPES, bem como, foram incluídos livros, comunicações oficiais e diretrizes de importância ao tema relacionado. Este tipo de revisão é apropriada para discutir o estado da arte de um determinado assunto. É constituída por uma análise ampla da literatura, sem estabelecer uma metodologia rigorosa e replicável em nível de reprodução de dados e respostas quantitativas para questões específicas, como explicitam Vosgerau e Romanowsk (2014). No entanto, é fundamental para a aquisição e atualização do conhecimento sobre uma temática específica, evidenciando novas ideias, métodos e subtemas que têm recebido maior ou menor ênfase na literatura selecionada (Elias et al., 2012).

Foram empregados os termos descritos no DeSC: Pequi, Diabetes Mellitus tipo 2, Inflamação e Estresse oxidativo, em português, inglês e espanhol, isolados ou de forma combinada, sem delimitar um intervalo temporal. O critério utilizado para inclusão das publicações era ter as expressões utilizadas nas buscas no título ou palavras-chave, ou ter explícito no resumo associação entre diabetes mellitus tipo 2 e pequi. Ademais, apenas foram incluídos nessa revisão estudos originais publicados no período até junho de 2021, que tratassem do tema em questão. Para a seleção dos artigos a serem utilizados nesse estudo inicialmente foi feita a leitura dos resumos e rejeitaram-se aqueles que não preencheram os critérios de inclusão e/ou apresentavam duplicidade, ou seja, publicações recuperadas em mais de uma das bases de dados.

\section{Resultados e Discussão}

\subsection{Diabetes mellitus tipo 2}

O diabetes mellitus (DM) abrange um grupo de doenças metabólicas e que tem em comum a hiperglicemia, resultante de defeitos na secreção de insulina ou de sua ação no organismo, está associada a várias complicações dentre as quais destacase disfunção e falência de vários órgãos. O aumento na incidência do diabetes tem se relacionado as mudanças de estilo de vida e do aumento no consumo de alimentos industrializados (Rossi, Silva \& Fonseca, 2015).

Esta condição é classificada etiologicamente em diabetes do tipo $1 \mathrm{em}$ que ocorre destruição da célula beta no pâncreas, podendo levar à deficiência completa de insulina, sendo necessária sua administração para evitar algumas complicações; O diabetes tipo 2 é uma das principais doenças crônicas que afetam o homem contemporâneo. Dentre fatores 
responsáveis, destacam-se: história familiar de DM2, urbanização, estilo de vida, dieta inadequada, sedentarismo, consumo de álcool e hipertensão arterial. O diabetes gestacional caracterizado como o aparecimento da hiperglicemia durante a gravidez em que normalmente regulariza no período pós parto; e outros tipos específicos de diabetes (Gomes \& Vidal, 2011; Magalhães et al., 2015).

Nos últimos anos o número de pessoas com DM2 tem aumentado e, por esse motivo, essa doença metabólica está se tornando um dos mais importantes desafios de saúde global (Singh et al., 2013). Em 2013, cerca de 382 milhões de adultos em todo o mundo tinham a doença, e é estimado para 2035 um aumento de $55 \%$ nos casos, com a maioria dos doentes na faixa etária entre 40 e 59 anos (Guariguata et al., 2014). Para o Brasil, o contingente estimado, passará de 11,9 milhões de casos, podendo alcançar 19,2 milhões em 2035 (International Diabetes Federation, 2014).

Segundo Carvalho et al. (2012), o aumento da prevalência do diabetes está relacionado ao envelhecimento da população, à prevalência crescente da obesidade, às mudanças no estilo de vida marcadas por aumento da ingestão de gorduras e açúcares e pelo sedentarismo. A hiperglicemia, frequentemente acompanhada de dislipidemia, anormalidades do metabolismo de lipoproteínas, obesidade abdominal, hipertensão arterial e disfunção endotelial, também compõem o quadro que caracteriza essa doença (Sociedade Brasileira de Diabetes, 2015). A associação desses fatores é determinante no desencadear de várias complicações que reduzem a esperança de vida dos doentes diabéticos, tais como doenças cardiovasculares, retinopatia, nefropatia e neuropatia (Ferreira et al., 2011; Souza et al., 2012).

A persistência do estado hiperglicêmico no diabético é o fator primário desencadeador de complicações macrovasculares, microvasculares e de distúrbios metabólicos. O aumento da concentração sérica de glicose aumenta a formação endógena dos produtos de glicação avançada (AGEs) circulantes, o que leva ao aumento dos níveis plasmáticos de apoproteína B (ApoB-AGE), que por ser constituinte da lipoproteína de baixa densidade (LDL), contribui para o desenvolvimento da aterosclerose por meio da deposição da LDL e ApoB-AGE na parede das artérias. Em decorrência disso, há comprometimento das artérias coronarianas, das artérias dos membros inferiores e das cerebrais, resultando em maior risco de doença arterial coronariana, doença vascular periférica e acidente vascular cerebral (Barbosa et al., 2009; Ferreira et al., 2011).

Os AGEs ligam-se às proteínas, aos lipídios e aos ácidos nucléicos, contribuindo para o surgimento de complicações do DM. Eles interagem também com receptores localizados na membrana plasmática para alterar a sinalização intracelular, a expressão gênica e a liberação de citocinas pró-inflamatórias, citocinas pró- aterogênicas e radicais livres, o que contribui ainda mais para o desenvolvimento e a progressão das complicações na doença (Bandeira et al., 2013; Singh et al., 2014).

Estudos comprovam que a hiperglicemia, em longo prazo, está associada com a glicação avançada, glicoxidação, estresse oxidativo e inflamação. Nesse sentido, o monitoramento e o controle da glicemia, são fundamentais para a prevenção de complicações e manutenção da qualidade de vida (Garcia et al., 2010; Navale \& Paranjape, 2013; Negre-Salvayre et al., 2009).

Entre as formas de tratamento do diabetes, além da terapia farmacológica, destacam-se a dieta e o exercício físico, sendo esses últimos de grande importância para o tratamento do DM2, porém devem ser empregadas em um plano individualizado e que requer a adesão do paciente. As principais mudanças alimentares necessárias envolvem a ingestão de alimentos com baixo índice glicêmico e ricos em fibras alimentares. A prática regular e orientada de exercício físico também tem importante papel do tratamento dessa doença, uma vez que ajuda na redução da glicação da hemoglobina, no aumento da sensibilidade dos tecidos à ação da insulina e na redução dos níveis circulantes de triglicérides e LDL (American Diabetes Association, 2014; Codogno, Fernandes \& Monteiro, 2012). 


\subsection{Diabetes mellitus tipo 2 e estresse oxidativo}

Estudos mostram a relação entre hiperglicemia e danos oxidativos aos lipídios, carboidratos, proteínas e ao DNA, em diferentes tecidos. Nesse sentido, a hiperglicemia crônica está relacionada à produção de espécies reativas de oxigênio (Karnakaran; Park, 2013). Os principais eventos metabólicos que explicam essa relação no interior das células estão associados ao aumento da atividade da via dos polióis; à ativação da Proteína Quinase C (PKC); e aumento da formação de produtos de glicação avançada, a partir da auto-oxidação da glicose (Barbosa et al., 2009; Giacco \& Brownlee, 2010; Yamagishi, 2011).

A indução do estresse oxidativo é um processo chave no aparecimento de complicações diabéticas (Kostic et al., 2009; Matough et al., 2012). Uma característica importante do estresse oxidativo em pacientes com diabetes está relacionada à manifestação da peroxidação lipídica. A resistência à insulina, presente nesses indivíduos, contribui para o aumento dos níveis séricos de triglicerídeos e de colesterol total; e essas moléculas são susceptíveis ao ataque das espécies reativas de oxigênio, o que leva ao dano oxidativo dessas lipoproteínas (Cimbaljevic et al., 2007).

A ação de EROS sobre os ácidos graxos poliinsaturados leva a uma cascata de eventos conhecida como peroxidação lipídica. Em pacientes com DM2, Bandeira et al. (2013) observaram um aumento da peroxidação lipídica, bem como uma estreita relação com altos níveis de glicose sanguínea em jejum e com o aumento na glicação da hemoglobina. Por outro lado, a ocorrência de peroxidação lipídica pode ser atribuída também à redução do consumo de nutrientes antioxidantes, redução da atividade das enzimas antioxidantes, e baixas concentrações plasmáticas e eritrocitárias de minerais, como o zinco, selênio e magnésio (Kostic et al., 2009; Pan et al., 2008).

No estudo de Begum et al. (2015) foi detectada precocemente a presença de complicações diabéticas através da determinação de marcadores de peroxidação lipídica, como a mieloperoxidase sérica (MPO), malondialdeído (MDA), bem como pela avaliação do perfil lipídico. No que diz respeito a esses marcadores, Kataoka et al. (2014) observaram que níveis crescentes de MPO estão associados com maior progressão da aterosclerose em pacientes diabéticos, o que indica a importância potencial de vias de MPO na doença cardiovascular diabética.

O estado pró-oxidante vem sendo relacionado com várias doenças crônicas, ora como causa e, em alguns casos, como consequência. Além dos fatores de risco clássicos, diversos biomarcadores têm sido relacionados ao estresse oxidativo, entre eles estão os marcadores de inflamação (proteína $\mathrm{C}$ reativa e ceruloplasmina), marcadores metabólicos (paraoxonase, adipocinas, homocisteína), bem como marcadores do "status" antioxidante, tais como enzimas antioxidantes (superóxido dismutase e glutationa peroxidase) e microelementos envolvidos com a atividade antioxidante (Vellosa et al., 2013). A avaliação do estresse oxidativo pode ser feita por meio de três abordagens, pela determinação de indicadores indiretos da atividade das EROS, avaliação direta das espécies reativas e determinação da concentração de antioxidantes (Reyes et al., 2006).

Com relação a esta última abordagem, os organismos possuem um sistema de defesa antioxidante constituído por componentes enzimáticos e não enzimáticos, que atuam conjuntamente na proteção celular. $\mathrm{O}$ sistema enzimático é considerado a primeira linha de defesa, uma vez que evita o acúmulo do radical ânion superóxido (O2-) e do peróxido de hidrogênio (H2O2), e é constituído principalmente pelas enzimas superóxido dismutase (SOD), catalase (CAT) e glutationa peroxidase (GPx) (Halliwell, 2006). A SOD é a primeira enzima na eliminação do ânion superóxido e a GPx é responsável por catalisar a redução do $\mathrm{H} 2 \mathrm{O} 2$ e de hidroperóxidos orgânicos (Rodriguez et al., 2004; Townsend et al., 2003). A formação de ânions superóxido e de peróxidos de hidrogênio ocorre pela auto-oxidação da glicose, causada pela hiperglicemia (Giacco \& Brownlee, 2010). Já o sistema não enzimático corresponde especialmente aos compostos obtidos da dieta, entre eles, as vitaminas antioxidantes A, C e E, e os minerais antioxidantes zinco, selênio e cobre (Barbosa et al., 2010; Carocho \& Ferreira, 2013; França et al., 2013). 
Os antioxidantes podem inibir a atividade de radicais livres através de vários mecanismos, incluindo sua participação nas enzimas que sequestram esses radicais e a sua capacidade de se ligarem a metais que estimulam a produção de radicais livres, inibindo assim, sua formação (Chaturvedi, 2007; Kaur \& Henry, 2014).

\subsection{Diabetes mellitus tipo 2 e inflamação}

Outra característica importante do DM2 é a presença de inflamação sistêmica crônica, em que fatores ambientais, metabólicos e genéticos estão envolvidos. Além disso, a inflamação está associada com o aumento do risco de desenvolver complicações (Garcia et al, 2010). No DM2 os componentes do sistema imune sofrem alterações, principalmente no tecido adiposo, fígado e ilhotas pancreáticas. Essas modificações constituem importantes alterações imunológicas na liberação de citocinas e quimiocinas específicas, no número de leucócitos e no aumento da apoptose e fibrose de tecidos (Donath \& Shoelson, 2011).

A inflamação ocorre como resposta a uma alteração da integridade do tecido, a fim de restaurar a homeostase por meio da indução de vários mecanismos de reparação (Goldszmid \& Trinchieri, 2012). Nos últimos anos foi comprovado que processos que envolvem reações redox desencadeantes de estresse oxidativo celular podem levar ao desenvolvimento do processo de inflamação (Liaudet et al., 2009; Nathan \& Cunningham-Bussel, 2013). Dessa forma, a inflamação consiste em um importante fator para o aumento do estado de hiperglicemia presente no diabetes, pois ela leva ao desenvolvimento de resistência à insulina. Entre os possíveis mecanismos ligados a esse processo estão as alterações nas vias de sinalização ligadas à produção de espécies reativas, e modificações na fosforilação de receptores de insulina, os IRS-1, resultando em interrupção do processo de sinalização (Carvalho et al., 2006; Newsholme \& Krause, 2012).

A resistência insulínica resulta em perturbação na homeostase de lipídios e citocinas, e na produção de adipocitocinas, resultando em aumento da inflamação, bem como em níveis mais elevados de marcadores inflamatórios, tais como, a Proteína C Reativa (PCR), Fator de Necrose Tumoral- $\alpha$ (TNF- $\alpha$ ) e Interleucina-6 (IL- 6) (Garcia et al., 2010; Pedicino et al., 2013). Vários estudos têm mostrado que a inflamação, mais especificamente a presença de citocinas pró-inflamatórias, é um fator determinante no desenvolvimento de complicações micro e macrovasculares no diabetes (Mora \& Navarro, 2006; Wu et al., 2012; Navale \& Paranjape, 2013).

Marcadores inflamatórios são proteínas de baixo peso molecular com funções metabólicas e endócrinas específicas, as quais participam dos mecanismos de inflamação e da resposta imunológica do organismo para garantir a homeostase. Eles podem ser divididos em: citocinas pró e anti-inflamatórias, quimiocinas, adipocinas, marcadores de inflamação de hepatócitos e enzimas (Poledne et al., 2009; Gottlieb et al., 2005; Volp et al. 2008). Os níveis séricos desses biomarcadores podem fornecer informações importantes sobre a inflamação e seus processos, incluindo a ativação das células vasculares e recrutamento de leucócitos e macrófagos (Libby, 2006).

A proteína quimioatrativa de monócitos - 1 (MCP-1) é uma quimiocina que controla a função dos monócitos por meio de seu receptor, e que está envolvida em mudanças inflamatórias na parede arterial, prejudicando a vasodilatação endotéliodependente. Além disso, desempenha um papel causal no recrutamento de leucócitos mononucleares para a camada íntima da artéria (Dod et al., 2010). Já a proteína $\mathrm{C}$ reativa (PCR) é considerada uma proteína inflamatória de fase aguda, sintetizada pelo fígado e regulada por citocinas, predominantemente a IL-6, o TNF- $\alpha$ e a IL-1. Elevações modestas dos níveis séricos de PCR estão também presentes em situações crônicas inflamatórias como a aterosclerose, e seus níveis aproximadamente triplicam na presença de risco de doenças vasculares periféricas. Uma de suas funções mais importantes é sua capacidade de se ligar aos componentes da membrana celular, formando complexos que ativam a via clássica, com liberação de opsoninas, com fagocitose e remoção dessas estruturas da circulação (Kinlay \& Selwyn, 2003; Alisson et al., 2012; Santos et al., 2008). 


\subsection{Diabetes mellitus tipo 2 e antioxidantes da dieta}

O efeito protetor dos antioxidantes tem sido mostrado em estudos clínicos, experimentais e epidemiológicos, os quais enfatizam que os antioxidantes podem ser úteis no tratamento do diabetes e suas complicações (Matough et al., 2012; Psaltopoulou et al., 2011; Rafigui et al., 2013). A ingestão de antioxidantes tem sido empregada como uma estratégia de intervenção para melhorar a longevidade de pacientes com DM2 (Ceriello, 2009). Os antioxidantes podem inibir a atividade de radicais livres através de vários mecanismos, incluindo sua participação nas enzimas que sequestram esses radicais e a sua capacidade de se ligarem a metais que estimulam a produção de radicais livres, inibindo assim, sua formação (Chaturvedi, 2007).

Os frutos das espécies nativas do Cerrado possuem atrativos sensoriais como cor, sabor e aroma muito peculiares e intensos, e são consumidos pela população local de forma in natura ou processados; observa-se também o uso de extratos de plantas nativas no tratamento ou prevenção de algumas enfermidades (Mendonça et al., 1998). Esses frutos podem apresentar um significativo potencial nutricional e de compostos bioativos com propriedades promotoras de saúde, que auxiliam na atividade antioxidante, sendo utilizados na medicina popular como antiinflamatórios, antibióticos, hipocolesterolêmicos (De Mesquita et al., 2009; Gonçalves, Lajolo \& Genovese, 2010). Essas atividades biológicas estão associadas a presença dos compostos bioativos, que promovem respostas fisiológicas capazes de modular o processo metabólico de inflamação, estresse oxidativo, atividades anti-hipertensiva, antimicrobiana e antimutagênica, e, consequentemente, reduzir o risco de doenças crônicas (Kaur et al., 2006; Lima et al., 2007; Denger et al., 2020).

Um estudo com frutos do Cerrado avaliou o potencial antioxidante nos extratos aquosos e etanólicos das polpas, semente e casca do araticum, lobeira, cagaita, pequi e banha de galinha. Foi encontrado alto potencial de redução de radicais livres na casca do pequi (Caryocar brasiliense; Caryocaraceae), semente de cagaita, semente e casca de araticum, e casca de banha de galinha (Swartizia Langs-dorffi), através da metodologia de DPPH (2,2-diphenyl-1-picrylhydrazyl). Embora os autores também tenham utilizado partes dos frutos que não são comestíveis, as mesmas precisam ser estudadas e avaliadas quanto a capacidade farmacológica de seus extratos (Roesler et al., 2007).

\subsection{Pequi (Caryocar Brasiliense Camb)}

O nome pequi é de origem tupi, em que py significa casca ou pele e qui corresponde a espinho, originando "casca espinhosa", isso devido aos espinhos do endocarpo lenhoso ou do caroço (Carvalho, 2008). O pequi (Caryocar brasiliense Camb.) é conhecido também, conforme o local de sua ocorrência, como almendro, amêndoa-de-espinho, amêndoa-do-Brasil, barbasco, grão de cavalo, pequerim, pequi-do-cerrado, pequiá, pequiá-pedra, pequiá- verdadeiro, pequiá- vermelho, piqui, piquiá, piqui-vinagreiro, piquiá-bravo, pitiá, sacode-bode ou suari (Carvalho, 2008; Ribeiro, 2000).

$\mathrm{O}$ fruto é do tipo drupa, com cerca de $5 \mathrm{a} 7 \mathrm{~cm}$ de diâmetro, podendo ultrapassar $10 \mathrm{~cm}$, cujo peso varia entre $30 \mathrm{~g} \mathrm{a}$ $400 \mathrm{~g}$ e possui de 1 a 6 pirênios por fruto (Carvalho, 2008). É constituído: pelo epicarpo, que possui coloração esverdeada ou marrom-esverdeada; pelo mesocarpo externo, rico em tanino, fibroso e de coloração verde clara ou levemente amarela; pelo mesocarpo interno, porção comestível do fruto que possui geralmente coloração amarelada, mas pode apresentar cor laranja, rósea ou esbranquiçada, e é pastosa e oleaginosa; e pelo endocarpo, parte espinhosa que protege a semente ou amêndoa. A semente, que também pode ser comestível, é revestida por um tegumento fino de coloração marrom (Carvalho, 2008; Ribeiro, 2000; Araújo, 1995). A casca deste fruto é responsável por aproximadamente $84 \%$ do peso do pequi, sendo que a polpa corresponde a 10\% e as sementes a 6\% (Barbosa \& Amante, 2002; Ferreira et al., 1988).

A polpa do fruto, parte mais importante em termos de utilização, possui alto valor energético, provindos de lipídios (Segal et al., 2006) e é onde se encontram teores médios de vitaminas A (retinol), B1 (tiamina), B2 (riboflavina), B3 (niacina) e C (ácido ascórbico) (Gonçalves et al., 2011; Almeida, Costa \& Silva, 2008; Franco, 1999; Sano \& Almeida, 1998). Há a 
presença de diversos carotenoides, porém somente alguns possuem atividade provitamina $\mathrm{A}$, tanto na polpa crua quanto cozida (Azevedo-Meleiro \& Rodriguez-Amaya, 2004; Ramos et al., 2001).

A polpa e a amêndoa deste fruto são fontes consideráveis de minerais como cálcio, cobre, ferro, fósforo, magnésio, manganês, potássio, sódio e zinco (Oliveira et al., 2010; Almeida \& Silva, 1994). Quanto às proteínas, os teores encontrados na polpa variam de $6,71 \%$ a $13,5 \%$; na amêndoa, variam de $24 \%$ a $54 \%$; e no óleo, de $42,2 \%$ a $47 \%$ (Oliveira et al., 2006). A polpa contém alto teor de fibra bruta, valores médios de carboidratos (Vilas Boas, 2004) e diversos compostos antioxidantes, como a vitamina C e os compostos fenólicos (Almeida, Costa \& Silva, 2008; Roesler et al., 2007; Oliveira et al., 2006). O óleo da polpa de pequi é constituído, principalmente, pelos ácidos oleico e palmítico, porém apresenta os ácidos palmitoléico, linoleico, esteárico, entre outros (Almeida, Costa \& Silva, 2008; Segal et al., 2006; Almeida, 1998).

O fruto é utilizado mais frequentemente na culinária, sendo raro o seu consumo "in natura". Sua polpa é utilizada na elaboração de diferentes pratos, como arroz com pequi, feijão com pequi, frango com pequi, cuscuz com pequi e o tradicional baião de três (arroz, feijão e pequi). Também é utilizado na produção de geleias, doces, bolos, farinhas, temperos (líquido e tabletes), cosméticos, sabão artesanal, sucos e sorvetes, ração para porcos e galinhas e na obtenção de óleo; a polpa fermentada produz um tipo de licor bastante conhecido e apreciado em algumas regiões brasileiras (Oliveira et al., 2008). Outra utilização da polpa quando fermentada é sua utilização na produção de xaropes para combater diversos tipos de afecções, principalmente as do sistema respiratório (Ramos et al., 2001; Almeida \& Silva, 1994).

O óleo é rico em ácido graxo oleico, vitaminas A e C, tiamina, proteínas e sais minerais, além de ótima qualidade para o consumo culinário (Perez, 2004). É utilizado na medicina popular, atuando contra bronquites, gripes e resfriados, além de possuir efeito tonificante. Usado, também, para sanar problemas oftalmológicos ligados a deficiência de vitamina A, comprovado cientificamente devido ao seu alto teor de carotenoides com atividade provitamina A (Almeida, Costa \& Silva, 2008; Oliveira et al., 2006, Brandão, Laca-Buendia \& Macedo, 2002; Ribeiro, 1996). É um dos ingredientes de cremes para pele na indústria de cosméticos e de sabões na de limpeza (Almeida et al., 1998). Tem potencial de uso na produção de combustíveis e lubrificantes (Oliveira et al., 2008).

Usa-se a amêndoa como ingrediente de farofas, doces e paçocas, além de ser consumida salgada como petisco (Ribeiro, 2000; Araújo, 1995). O pequi é pouco industrializado, sendo explorado, ainda, de forma artesanal. A extração do óleo dos frutos é sua principal forma de processamento. A conserva de pequi, que vem se destacando nas regiões produtoras, recentemente exportada, e a fabricação do licor são outros tipos de processamento (Vieira et al.,2010).

Por se tratar de um fruto de fácil produção e com boas características quanto ao sabor e valor nutritivo, pode representar uma potencial fonte na alimentação e sobrevivência de uma parcela da população brasileira (Ribeiro, 2000; Araújo, 1995). Exerce grande influência na renda dos agricultores habitantes do Cerrado, além de ter importante papel na sua alimentação, sendo muito consumido pelas populações nativas em que são encontrados, assim, om a demanda por pequis na época de safra, muitos coletores os derrubam direto da árvore ("pequi de vara"), sem que o processo de maturação esteja totalmente completo afetando a qualidade dos frutos, sendo esse um dos principais problemas do extrativismo atual do pequi (Brait, 2008).

Devido ao processo de período de floração, frutificação e maturação do pequi ser de aproximadamente três meses, existe, assim, uma carência desse fruto na entressafra. Dessa forma, para dispor dessa espécie na entressafra, são desenvolvidos processos de produção, de beneficiamento e de comercialização de diversos produtos desse fruto, tais como polpa congelada e diversos tipos de pequi em conserva. Ações dessa natureza agregam cerca de 50\% do valor em relação ao produto in natura (Oliveira et al., 2008). 


\subsection{Compostos bioativos e potencial antioxidante da polpa de pequi}

Estudos acerca dos teores de compostos bioativos em frutos nativos do Cerrado, considerando a biodiversidade desse bioma, são escassos, ou, quando existentes, muitos estão delineados de forma inadequada (Azevedo-Meleiro \& RodriguezAmaya, 2004). Barreto, Benassi e Mercadante (2009) investigaram a presença de compostos bioativos (ácido ascórbico, compostos fenólicos totais, flavonoides totais e carotenoides totais) e a atividade antioxidante de vários frutos regionais, provenientes de Manaus-AM, Fortaleza-CE e Campinas-SP. Seus resultados mostraram que os frutos com maiores teores de compostos bioativos foram: buriti, cajá-manga, canistel, murici, physalis, pequi e tucumã. A atividade antioxidante apresentou alta correlação com os teores de flavonoides e compostos fenólicos totais. Há, porém, uma limitação considerável neste estudo, já que não são especificadas as espécies e nem a localização específica onde foram coletados ou adquiridos os frutos.

Em relação ao conteúdo de carotenoides em alimentos regionais, como o pequi (Caryocar brasiliense Camb.), Lima et al. (2007) encontraram elevadas quantidades de carotenoides totais $(7,25 \mathrm{mg} .100 \mathrm{~g}-1)$ na polpa de frutos de pequi procedentes do estado do Piauí. Em estudo feito por Azevedo-Meleiro e Rodriguez-Amaya (2004) foram identificados, por cromatografia líquida de alta performance (HPLC - High Performance Liquid Cromatography), os carotenoides presentes na polpa de frutos de pequis (Caryocar brasiliense) provenientes de Belo Horizonte-MG. Seus resultados revelaram que os principais carotenoides presentes na polpa deste fruto foram violaxantina, luteína e zeaxantina. Ramos et al. (2001) também investigaram qualitativamente o teor de carotenoides em pequis, oriundos de Campo Grande-MS, porém por cromatografia em coluna aberta. Neste estudo foi constatado que a anteraxantina era o pigmento carotenoide predominante na polpa do pequi.

O estudo realizado por Denger et al. (2020) indicaram que o extrato aquoso de pequi, na proporção testada $4 \mathrm{~g} / \mathrm{dl}$, exibiu forte atividade antioxidante in vitro e ex vivo, variável conforme a metodologia utilizada, ratificando o pequi como u ma matéria-prima de potencial antioxidante natural, com propriedades comerciais, que lança luz à possíveis aplicações tecnológicas e funcionais exploráveis, além de consequente ampliação dos seus usos, favorecendo o desenvolvimento econômico e social. Nesse estudo o conteúdo fenólico total foi de $2,22 \pm 0,045 \mathrm{~g}$ de equivalentes de ácido tânico/100 $\mathrm{g}$ e o teste de DPPH evidenciou que todas as frações das amostras processadas possuíram atividade em suas diferentes concentrações. Em relação a inibição da peroxidação lipídica, os resultados demonstraram redução dos níveis, existindo diferença significativa ( $\mathrm{p}<$ a partir da concentração de $0,02 \mathrm{~g} / \mathrm{dl}$.

$\mathrm{Na}$ literatura não há estudos que tenham investigado as diferenças entre o teor e o perfil de carotenoides de pequis provenientes das diversas regiões do Cerrado, considerando sua biodiversidade. Portanto, há necessidade de mais pesquisas científicas tendo os frutos regionais brasileiros, especialmente o pequi, como foco de estudo para determinação de carotenoides, utilizando metodologias padronizadas, e que relacionem o teor de carotenoides com as características físicas e procedência dos frutos. Já que a coloração da polpa de pequi é conferida pelos pigmentos carotenoides, seria interessante a realização de pesquisas que associassem essas duas características.

Quanto ao teor de compostos fenólicos totais da polpa de pequi, Lima et al. (2007), em estudo com pequis oriundos do Piauí, constataram teores consideráveis para a polpa desse fruto (209 mg.100-1g). Foram encontrados na literatura poucos estudos que descrevesse o conteúdo de compostos bioativos em pequis nativos dos estados que compõem o Cerrado brasileiro. Um trabalho feito por Roesler et al. (2007), cujo objetivo era quantificar os compostos fenólicos totais e avaliar o potencial antioxidante de alguns frutos do Cerrado, mostrou que a polpa e semente do pequi não apresentaram boa quantidade de fenóis quando comparadas com a casca deste fruto, nem apresentaram um bom potencial antioxidante. É importante considerar que este estudo foi realizado com frutos de apenas uma região de Goiás (Goiânia), não considerando as outras regiões do Cerrado brasileiro, que possuem frutos de pequi com diferenças morfológicas significativas, conforme mostrado nos estudos de Vera et al., (2005 e 2007). 
Há, ainda, estudos mostrando que o óleo da polpa de pequi, proveniente de Brasília-DF, foi eficiente para reduzir danos teciduais em atletas corredores, sugerindo seu uso como suplemento antioxidante (Miranda-Vilela et al., 2009a; Miranda- Vilela et al., 2009b). É importante considerar que pode haver diferenças na composição química dos frutos de pequi originários de diferentes regiões do Cerrado, o que pode interferir significativamente no seu potencial antioxidante. Em outro estudo realizado por Sousa et al. (2012) utilizando capsulas de $400 \mathrm{mg}$ óleo de pequi por 60 dias, foi demonstrado efeito positivo do OAP em reduzir a produção de EROS em pacientes portadores de Lúpus Eritematoso Sistêmico (LES). No entanto não há na literatura dados que relacionem especificamente o uso do OAP e o DM2.

Ensaios pré-clínicos do óleo fixo da polpa do Caryocar coriaceum (OFCC) apontam a atividade gastroprotetora em roedores com significativa redução de úlceras induzidas por etanol e aspirina (Penha, 2007; Quirino et al., 2009) e cicatrizante (Quirino et al., 2009). O óleo fixo também possui efeito anti-inflamatório tópico em camundongos, e demonstrou eficácia na redução de inflamação cutânea, com tratamento crônico (Saraiva, 2009).

O óleo fixo da polpa do Caryocar coriaceum (OFCC) tem como componente majoritário o ácido graxo insaturado oléico. Os ácidos graxos saturados aumentam a lipoproteína de baixa densidade (LDL) por inibição da atividade do receptor de LDL e aumentam a produção de apolipoproteína. A substituição de gordura saturada por gordura poliinsaturada tem reduzido os níveis de colesterol total (CT) e o LDL colesterol, por diminuição das taxas de produção de LDL colesterol e/ou aumento das taxas de depuração de LDL. Em contrapartida, há diminuição da lipoproteína de alta densidade (HDL), que juntamente com a redução do LDL-c, diminuem a razão LDL / HDL. Enquanto, que a gordura monoinsaturada também tem o mesmo efeito na colesterolemia, mas a magnitude da redução de HDL é menor quando comparada as gorduras poliinsaturadas (Aguilar et al., 2012).

Sabe-se que a oferta e diversidade de alimentos é indispensável para combater a má nutrição e as enfermidades em populações vulneráveis em um contexto mundial de crescimento populacional. Embora sejam crescentes as pesquisas sobre frutos do Cerrado, ainda não há estudos suficientes que comprovem a atividade biológica e descrevam os compostos responsáveis por essas propriedades, o que reforça a importância das pesquisas nesta área. Sugere-se que os frutos do Cerrado podem apresentar grande quantidade e diversidade de compostos bioativos, e que estes também podem oferecer benéficos para a saúde do indivíduo, representando uma potencial fonte de alimentos com propriedades funcionais a ser incorporada na alimentação da população, ou ainda, serem utilizados na indústria farmacêutica e de cosméticos.

\section{Conclusão}

Diante das alterações metabólicas e bioquímicas normalmente verificadas no diabetes mellitus tipo 2 envolvendo a produção excessiva de espécies reativas de oxigênio e distúrbios na atividade de enzimas antioxidantes e marcadores de inflamação, pode-se inferir a partir dos dados aqui expostos que o consumo de alimentos fontes de antioxidantes como frutos do cerrado, especificamente o pequi podem contribuir para prevenção dessa cascata patológica presente no DM2, no entanto são necessários a realização de mais estudos in vivo e de intervenção que possam contribuir para um melhor entendimento acerca da influência de compostos antioxidantes presentes no óleo de pequi sobre o sistema de defesa antioxidante e atividade inflamatória na presença de hiperglicemia crônica.

\section{Referências}

Aguilar, E. C., Jascolka, T. L., Teixeira, L. G., Lages, P. C., Ribeiro, A. C. C., Vieira, E. L. M., \& Alvarez-Leite, J. I. (2012). Paradoxical effect of a pequi oilrich diet on the development of atherosclerosis: balance between antioxidant and hyperlipidemic properties. Brazilian Journal of Medical and Biological Research, 45, 601-609.

Allison, M. A., Jensky, N. E., Marshall, S. J., Bertoni, A. G., \& Cushman, M. (2012). Sedentary behavior and adiposity-associated inflammation: the MultiEthnic Study of Atherosclerosis. American journal of preventive medicine, 42(1), 8-13. 
Almeida, S. D., \& Agostini-Costa, T. S. (1998). Frutas nativas do cerrado: caracterização físico-química e fonte potencial de nutrientes. Cerrado: ambiente e flora. In: Sano, S. M.; Almeida, S. P. Cerrado: ambiente e flora. Planaltina: EMBARAPA-CPAC, 1998. 247-285.

Alves, C. C. D. O., Resende, J. V. D., Cruvinel, R. S. R., \& Prado, M. E. T. (2008). Estabilidade da microestrutura e do teor de carotenóides de pós obtidos da polpa de pequi (Caryocar brasiliense Camb.) liofilizada. Food Science and Technology, 28, 830-839.

American Diabetes Association. (2014). Diagnosis and classification of diabetes mellitus. Diabetes care, 28(S37), S5-S10.

Araújo, J. (2004). Oxidação de lipídios em alimentos. In: Araújo, Jma Química de alimentos: teoria e prática, 3, 1-64.

Atlas, D. (2015). International diabetes federation. IDF Diabetes Atlas, 7th edn. Brussels, Belgium: International Diabetes Federation.

Azevedo-Meleiro, C. H., \& Rodriguez-Amaya, D. B. (2004). Confirmation of the identity of the carotenoids of tropical fruits by HPLC-DAD and HPLCMS. Journal of food Composition and Analysis, 17(3-4), 385-396.

Badawi, U., Klip, A., Haddad, P. (2010). Diabetes mellitus tipo 2 e inflamação: perspectivas de biomarcadores de risco e de intervenção nutricional. Diabetes Obes. Metab. Syndr, 3(1), 173-186.

Bandeira, D. M., Da Fonseca, L. J. S., Guedes, D. S., Rabelo, L. A., Goulart, M. O., \& Vasconcelos, S. M. L. (2013). Oxidative stress as an underlying contributor in the development of chronic complications in diabetes mellitus. International journal of molecular sciences, 14(2), 3265-3284.

Barbosa, J. H. P., Oliveira, S. L. D., \& Seara, L. T. (2009). Produtos da glicação avançada dietéticos e as complicações crônicas do diabetes. Revista de Nutrição, 22, 113-124.

Barbosa, Kiriaque Barra Ferreira, Costa, Neuza Maria Brunoro, Alfenas, Rita de Cássia Gonçalves, Paula, Sérgio Oliveira De, Minin, Valéria Paula Rodrigues, Bressan, Josefina. (2010). Estresse oxidativo: conceito, implicações e fatores modulatórios. Revista de Nutrição, $23(4)$ : 629-643.

Barbosa, R. C. M. V., Amante, E. R. (2002). Farinha de casca de pequi (Caryocar brasiliense). Sociedade Brasileira de Fruticultura.

Barreto, G. P., Benassi, M. T., \& Mercadante, A. Z. (2009). Bioactive compounds from several tropical fruits and correlation by multivariate analysis to free radical scavenger activity. Journal of the Brazilian Chemical Society, 20, 1856-1861.

Begum, M., Kumar, J. A., D’Souza, H. P., Sushith, S., Prathima, M. B., Shridhar, R., \& Kumar, K. A. (2015). Myeloperoxidase, malondialdehyde and serum lipids in type 2 diabetes mellitus. TC ( $\mathrm{mg} / \mathrm{dl}), 163(274), 0-001$.

Bernardes, T. G., Naves, R. V., Rezende, C. F. A., Borges, J. D., \& Chaves, L. J. (2008). Propagação sexuada do pequizeiro (Caryocar brasiliense Camb.) estimulada por ácido giberélico. Pesquisa Agropecuária Tropical, 38(2), 71-77.

Brait, J. D. A. (2008). Características físicas e químicas de pequizeiro (Caryocar brasiliense CAMB), de ocorrência natural em Rio Verde - GO. Dissertação (Mestrado em Agronomia) - Campus de Jataí, Universidade Federal de Goiás.

Brandão, M., de Minas, E. D. P. A., Laca-Buendia, J. P., \& Macedo, J. F. (2002). Árvores nativas e exóticas do Estado de Minas Gerais.

Carocho, M., \& Ferreira, I. C. (2013). A review on antioxidants, prooxidants and related controversy: natural and synthetic compounds, screening and analysis methodologies and future perspectives. Food and chemical toxicology, 51, 15-25.

Carvalho, F. S., Pimazoni Netto, A., Zach, P., Sachs, A., \& Zanella, M. T. (2012). Importância da orientação nutricional e do teor de fibras da dieta no controle glicêmico de pacientes diabéticos tipo 2 sob intervenção educacional intensiva. Arquivos Brasileiros de Endocrinologia \& Metabologia, 56(2), $110-119$.

Carvalho, M. H. C. D., Colaço, A. L., \& Fortes, Z. B. (2006). Cytokines, endothelial dysfunction, and insulin resistance. Arquivos Brasileiros de Endocrinologia \& Metabologia, 50(2), 304-312.

Carvalho, P. E. R. (2003). Espécies arbóreas brasileiras (1, 745-749). Embrapa Informação Tecnológica.

Chaturvedi, N. (2007). The burden of diabetes and its complications: trends and implications for intervention. Diabetes research and clinical practice, 76(3), S3-S12.

Cimbaljević, B., Vasilijević, A., Cimbaljević, S., Buzadžić, B., Korać, A., Petrović, V., \& Korać, B. (2007). Interrelationship of antioxidative status, lipid peroxidation, and lipid profile in insulin-dependent and non-insulin-dependent diabetic patients. Canadian journal of physiology and pharmacology, $85(10)$, 997-1003.

Ceriello, A. (2009). Hypothesis: the "metabolic memory", the new challenge of diabetes. Diabetes research and clinical practice, 86, S2-S6.

Codogno, J. S., Fernandes, R. A., \& Monteiro, H. L. (2012). Prática de atividades físicas e custo do tratamento ambulatorial de diabéticos tipo 2 atendidos em unidade básica de saúde. Arquivos Brasileiros de Endocrinologia \& Metabologia, 56, 06-11.

Cruz, N. G., Sousa, L. P., Sousa, M. O., Pietrani, N. T., Fernandes, A. P., \& Gomes, K. B. (2013). The linkage between inflammation and Type 2 diabetes mellitus. Diabetes research and clinical practice, $99(2), 85-92$.

Denger, A. P. F. L., Kawano, L. de O., Paula, R. A. de O., Santos, L. B., Rodrigues, M. R., Paula, F. B. de A., Duarte, S. M. da S. (2020). Determination of antioxidant activity and total phenols of pequi (Caryocar brasiliense Camb.). Research, Society and Development, 9(11).

De Almeida, S. P., \& Da Silva, J. A. (1994). Piqui e buriti: importância alimentar para a população dos cerrados. Embrapa Cerrados-Documentos (INFOTECA-E). In: Sano, S. M.; Almeida, S. P.; \& Ribeiro, J. F. (Ed.). Cerrado: ecologia e flora. EMBRAPA Cerrados, 2008. 1, 351-381 
De Oliveira L. G., Penha, A. R. S., da Silva, G. Q., Colares, A. V., Rodrigues, F. F. G., Costa, J. G. M., \& Campos, A. R. (2009). Gastroprotective effect of medicinal plants from Chapada do Araripe, Brazil. J Young Pharm, 1(1), 54.

De Oliveira Sousa, A. G., Fernandes, D. C., Alves, A. M., de Freitas, J. B., \& Naves, M. M. V. (2011). Nutritional quality and protein value of exotic almonds and nut from the Brazilian Savanna compared to peanut. Food Research International, 44(7), 2319-2325.

De Oliveira, M. L. M., Nunes-Pinheiro, D. C. S., Tomé, A. R., Mota, É. F., Lima-Verde, I. A., de Melo Pinheiro, F. G., \& De Morais, S. M. (2010). In vivo topical anti-inflammatory and wound healing activities of the fixed oil of Caryocar coriaceum Wittm. seeds. Journal of Ethnopharmacology, 129(2), 214-219.

De Oliveira, M. E. B., Guerra, N. B., Barros, L. D. M., \& Alves, R. E. (2008). Aspectos agronômicos e de qualidade do pequi. Embrapa Agroindústria Tropical-Documentos (INFOTECA-E).

Dod, H. S., Bhardwaj, R., Sajja, V., Weidner, G., Hobbs, G. R., Konat, G. W., \& Jain, A. C. (2010). Effect of intensive lifestyle changes on endothelial function and on inflammatory markers of atherosclerosis. The American journal of cardiology, 105(3), 362-367.

Donath, M. Y., \& Shoelson, S. E. (2011). Type 2 diabetes as an inflammatory disease. Nature reviews immunology, 11(2), 98-107.

Elias, C. S. R., Silva, L. A., Martins, M. T. S. L., Ramos, N. A. P. R., Souza, M. G. G. \& Hipólito, R. L. (2012) Quando chega o fim? Uma revisão narrativa sobre terminalidade do período escolar para alunos deficientes mentais. SMAD: Revista Electrónica em Salud Mental, Alcohol y Drogas, (8)1, 48-53.

Ferreira, I. M. (2008). Paisagens do Cerrado: um estudo do subsistema de Veredas. Universo do Cerrado. Goiânia: UCG, 1, 79-164.

Ferreira, L. T., Saviolli, I. H., Valenti, V. E., \& de Abreu, L. C. (2011). Diabetes melito: hiperglicemia crônica e suas complicações. Arquivos Brasileiros de Ciências da Saúde, 36(3).

França, B. K., Alves, M. R. M., Souto, F. M. S., Tiziane, L., Boaventura, R. F., Guimarães, A., \& Alves Jr, A. (2013). Peroxidação lipídica e obesidade: Métodos para aferição do estresse oxidativo em obesos. GE jornal português de gastrenterologia, 20(5), $199-206$.

Franco, G. (1995). Tabela de composiçäo química dos alimentos. In Tabela de composiçäo química dos alimentos (pp. 307-307).

Garcia, C., Feve, B., Ferre, P., Halimi, S., Baizri, H., Bordier, L., Mayaudon, H. (2010). Diabetes and inflammation: fundamental aspects and clinical implications. Diabetes \& metabolism, 36(5), 327-338.

Garcia-Bailo, B., El-Sohemy, A., Haddad, P. S., Arora, P., BenZaied, F., Karmali, M., \& Badawi, A. (2011). Vitamins D, C, and E in the prevention of type 2 diabetes mellitus: modulation of inflammation and oxidative stress. Biologics: targets \& therapy, $5,7$.

Giacco, F., \& Brownlee, M. (2010). Oxidative stress and diabetic complications. Circulation research, 107(9), 1058-1070.

Goldszmid, R. S., \& Trinchieri, G. (2012). The price of immunity. Nature immunology, 13(10), 932-938.

Gomes, C. M., \& Vidal, E. C. F. (2011). Idosos com diabetes mellitus: práticas de autocuidado. Cadernos de Cultura e Ciência, 10(2), $24-32$.

Gonçalves, G. A. S., Vilas Boas, E. V. D. B., Resende, J. V. D., Machado, A. L. D. L., \& Vilas Boas, B. M. (2011). Qualidade dos frutos do pequizeiro submetidos a diferentes tempos de cozimento. Ciência e Agrotecnologia, 35, 377-385.

Gottlieb, M. G., Bonardi, G., \& Moriguchi, E. H. (2005). Fisiopatologia e aspectos inflamatórios da aterosclerose. Scientia Medica, 15 (3), $203-7$.

Guariguata, L., Whiting, D. R., Hambleton, I., Beagley, J., Linnenkamp, U., \& Shaw, J. E. (2014). Global estimates of diabetes prevalence for 2013 and projections for 2035. Diabetes research and clinical practice, 103(2), 137-149.

Halliwell, B. (2006). Reactive species and antioxidants. Redox biology is a fundamental theme of aerobic life. Plant physiology, 141(2), 312-322.

Johnson, E. L. (2013). Glycemic Variability in Type 2 Diabetes Mellitus. Diabetes, 139-154.

Karunakaran, U., \& Park, K. G. (2013). A systematic review of oxidative stress and safety of antioxidants in diabetes: focus on islets and their defense. Diabetes \& metabolism journal, 37(2), 106-112.

Kataoka, Y. (2014). Níveis de mieloperoxidase prever acelerada progressão da aterosclerose coronária em pacientes diabéticos: Insights de ultrasom intravascular. Atherosclerosis, 232(2), 377-383.

Kaur, B., \& Henry, J. (2014). Micronutrient status in type 2 diabetes: a review. Advances in food and nutrition research, 71, 55-100.

Kinlay, S., \& Selwyn, A. P. (2003). Effects of statins on inflammation in patients with acute and chronic coronary syndromes. The American journal of cardiology, 91(4), 9-13.

Kostić, N., Čaparević, Z., Marina, Đ., Ilić, S., Radojković, J., Ćosić, Z., \& Bakić-Ćelić, V. (2009). Clinical evaluation of oxidative stress in patients with diabetes mellitus type II: Impact of acute exercise. Vojnosanitetski pregled, 66(6), 459-464.

Liaudet, L., Vassalli, G., \& Pacher, P. (2009). Role of peroxynitrite in the redox regulation of cell signal transduction pathways. Frontiers in bioscience: a journal and virtual library, 14, 4809.

Libby, P. (2006). Inflammation and cardiovascular disease mechanisms. The American journal of clinical nutrition, 83(2), 456S-460S.

Lima, A. D., Silva, A. M. D. O., Trindade, R. A., Torres, R. P., \& Mancini-Filho, J. O. R. G. E. (2007). Composição química e compostos bioativos presentes na polpa e na amêndoa do pequi (Caryocar brasiliense, Camb.). Revista Brasileira de Fruticultura, 29, 695-698. 
Lugrin, J., Rosenblatt-Velin, N., Parapanov, R., \& Liaudet, L. (2014). The role of oxidative stress during inflammatory processes. Biological chemistry, 395(2), 203-230.

Magalhães, A. T., da Silva, B. A. K., Ribeiro, J. A., de Aguiar Bisneto, J. F., Pereira, L. P. I., Machado, N. V., ... \& Cardoso, V. S. (2015). Avaliação do risco de desenvolver diabetes mellitus tipo 2 em população universitária. Revista Brasileira em Promoção da Saúde, 28(1), 5-15.

Mata, S. J. R. (2009). Extração e caracterização do óleo da amêndoa do pequi (Caryocar brasiliense Camb.) para uso em cosméticos. Brazilian Association of Chemistry.

Matough, F. A., Budin, S. B., Hamid, Z. A., Alwahaibi, N., \& Mohamed, J. (2012). The role of oxidative stress and antioxidants in diabetic complications. Sultan Qaboos University Medical Journal, 12(1), 5.

Ministério Da Saúde. (2006). http://www.portaldasaude.pt/portal/conteudos/enciclopedia+da+saude/minister osaude/doencas/doencas+cronicas doenças+aparelho+circulatorio/doencascardiovasculares.htm 2006.

Miranda-Vilela, A. L., Grisolia, C. K., Resck, I. S., \& Mendonça, M. A. (2009). Characterization of the major nutritional components of Caryocar brasiliense fruit pulp by NMR spectroscopy. Química Nova, 32, 2310-2313.

Miranda-Vilela, A. L., Pereira, L. C., Gonçalves, C. A., \& Grisolia, C. K. (2009). Pequi fruit (Caryocar brasiliense Camb.) pulp oil reduces exercise-induced inflammatory markers and blood pressure of male and female runners. Nutrition research, 29(12), 850-858.

Miranda-Vilela, A. L., Akimoto, A. K., Alves, P. C., Pereira, L. C., Gonçalves, C. A., Klautau-Guimarães, M. N., \& Grisolia, C. K. (2009). Dietary carotenoid-rich pequi oil reduces plasma lipid peroxidation and DNA damage in runners and evidence for an association with MnSOD genetic variantVa19Ala. Genet Mol Res, 8(4), 1481-95.

Montane, J., Cadavez, L., \& Novials, A. (2014). Stress and the inflammatory process: a major cause of pancreatic cell death in type 2 diabetes. Diabetes, metabolic syndrome and obesity: targets and therapy, 7,25.

Mora, C., \& Navarro, J. F. (2006). Inflammation and diabetic nephropathy. Current diabetes reports, 6(6), 463-468.

Nathan, C., \& Cunningham-Bussel, A. (2013). Beyond oxidative stress: an immunologist's guide to reactive oxygen species. Nature Reviews Immunology, 13(5), 349-361.

Navale, A. M., \& Paranjape, A. N. (2013). Role of inflammation in development of diabetic complications and commonly used inflammatory markers with respect to diabetic complications. Int J Pharm Pharm Sci, 5(Suppl 2), 1-5.

Negre-Salvayre, A., Salvayre, R., Augé, N., Pamplona, R., \& Portero-Otin, M. (2009). Hyperglycemia and glycation in diabetic complications. Antioxidants \& redox signaling, 11(12), 3071-3109.

Newsholme, P., Cruzat, V., Arfuso, F., \& Keane, K. (2014). Nutrient regulation of insulin secretion and action. Journal of Endocrinology, 221(3), R105-R120.

Tecnológica, E. I. (2006). Plano Nacional de Agroenergia.

Oliveira, M. E. B. D., Guerra, N. B., Maia, A. D. H. N., Alves, R. E., Matos, N. M. D. S., Sampaio, F. G. M., \& Lopes, M. M. T. (2010). Características químicas e físico-químicas de pequis da Chapada do Araripe, Ceará. Revista Brasileira de fruticultura, 32, 114-125.

Oliveira, M. N. S. D., Gusmão, E., Lopes, P. S. N., Simões, M. O. M., Ribeiro, L. M., \& Dias, B. A. S. (2006). Estádio de maturação dos frutos e fatores relacionados aos aspectos nutritivos e de textura da polpa de pequi (Caryocar brasiliense Camb.). Revista Brasileira de Fruticultura, 28(3), 380-386.

Pan, H. Z., Zhang, H., Chang, D., Li, H., \& Sui, H. (2008). The change of oxidative stress products in diabetes mellitus and diabetic retinopathy. British Journal of Ophthalmology, 92(4), 548-551.

Paneni, F., Beckman, J. A., Creager, M. A., \& Cosentino, F. (2013). Diabetes and vascular disease: pathophysiology, clinical consequences, and medical therapy: part I. European heart journal, 34(31), 2436-2443.

Passos, X. S., Castro, A. C. M., Pires, J. S., Garcia, A. C. F., Campos, F. C., Fernandes, O. F., \& Silva, M. D. R. R. (2003). Composition and antifungal activity of the essential oils of Caryocar brasiliensis. Pharmaceutical biology, 41(5), 319-324.

Pedicino, D., Liuzzo, G., Trotta, F., Giglio, A. F., Giubilato, S., Martini, F., \& Crea, F. (2013). Adaptive immunity, inflammation, and cardiovascular complications in type 1 and type 2 diabetes mellitus. Journal of diabetes research, 2013.

Perez, E. (2004). Diagnose fitoquímica dos frutos de Caryocar brasiliense Camb. Caryocaraceae [dissertation]. University of Paraná, Curitiba .

Penha, A. R. S. (2007). Estudo de atividade antiulcerogênica de plantas da chapada do Araripe. Monografia Graduação em Ciências Biológicas. Universidade Regional do Cariri, Crato

Pitocco, D., Tesauro, M., Alessandro, R., Ghirlanda, G., \& Cardillo, C. (2013). Oxidative stress in diabetes: implications for vascular and other complications. International journal of molecular sciences, 14(11), 21525-21550.

Poledne, R., Lorenzova, A., Stavek, P., Valenta, Z., Hubáček, J., Suchánek, P., \& Pit'ha, J. (2009). Proinflammatory status, genetics and atherosclerosis. Physiological research, 58(Suppl. 2), S111-S118.

Psaltopoulou, T., Panagiotakos, D. B., Pitsavos, C., Chrysochoou, C., Detopoulou, P., Skoumas, J., \& Stefanadis, C. (2011). Dietary antioxidant capacity is inversely associated with diabetes biomarkers: the ATTICA study. Nutrition, Metabolism and Cardiovascular Diseases, 21(8), 561-567.

Quirino, G. L., Gerlânia, R., Luciana, T. A., Costa, J. G., \& Herzog, A., \& Campos, A. (2009). Healing potential of Pequi (Caryocar coriaceum Wittm.) fruit pulp oil. Phytochemistry Letters. 2. 179-183. 10.1016/j.phytol.2009.06.002. 
Rabêlo, A. M. D. S., Torres, C. L., Geraldine, R. M., \& Silveira, M. F. A. (2008). Extração, secagem e torrefação da amêndoa do pequi (Caryocar brasiliense Camb.). Food Science and Technology, 28, 868-871.

Rafighi, Z., Shiva, A., Arab, S., \& Yusuf, R. M. (2013). Association of dietary vitamin C and E intake and antioxidant enzymes in type 2 diabetes mellitus patients. Global journal of health science, $5(3), 183$.

Ramadan, M. F., Asker, M. M. S., \& Tadros, M. (2012). Antiradical and antimicrobial properties of cold-pressed black cumin and cumin oils. European Food Research and Technology, 234(5), 833-844.

Ramos, M. I. L., Umaki, M. C. S., Hiane, P. A., \& Ramos Filho, M. M. (2001). Efeito do cozimento convencional sobre os carotenóides pró-vitamínicos" A" da polpa do piqui (Caryocar brasiliense Camb). Boletim do Centro de Pesquisa de Processamento de Alimentos, 19(1).

Rani, A. J., \& Mythili, S. (2014). Study on total antioxidant status in relation to oxidative stress in type 2 diabetes mellitus. Journal of clinical and diagnostic research: JCDR, 8(3), 108 .

Reyes, G. C., Sánchez, I. R., Calzada-Mendoza, C. C., \& Olivares-Corichi, I. M. (2006). Disfunción endotelial y estrés oxidativo. Rev Endocrinol Nutr, 14(4), 233-6.

Ribeiro, A. (1996). O espaço, o homem e o seu destino no norte de Minas. Universidade Federal De Lavras. Departamento de Administração e Economia. Manejo sustentado do Cerrado para uso múltiplo: subprojeto agroecologia e desenvolvimento. Lavras: UFLa, 11-18.

Ribeiro, M. C., Vilas Boas, E. V. D. B., Riul, T. R., Pantoja, L., Marinho, H. A., \& Santos, A. S. D. (2012). Influence of the extraction method and storage time on the physicochemical properties and carotenoid levels of pequi (Caryocar brasiliense Camb.) oil. Food Science and Technology, 32, 386-392.

Ribeiro, R. F. (2000). Pequi: o rei do cerrado. Rede Cerrado, 62. Rede Cerrado.

Rodriguez, C., Mayo, J. C., Sainz, R. M., Antolín, I., Herrera, F., Martín, V., \& Reiter, R. J. (2004). Regulation of antioxidant enzymes: a significant role for melatonin. Journal of pineal research, 36(1), 1-9.

Rossi, V. E. C., da Silva, A. L., \& Fonseca, G. S. S. (2015). Adesão ao tratamento medicamentoso entre pessoas com diabetes mellitus tipo 2. Revista de Enfermagem do Centro-Oeste Mineiro, 5(3).

Roesler, R., Malta, L. G., Carrasco, L. C., Holanda, R. B., Sousa, C. A. S., \& Pastore, G. M. (2007). Atividade antioxidante de frutas do cerrado. Food Science and Technology, 27(1), 53-60.

Santos, M. G. D., Pegoraro, M., Sandrini, F., \& Macuco, E. C. (2008). Fatores de risco no desenvolvimento da aterosclerose na infância e adolescência. Arquivos Brasileiros de Cardiologia, 90, 301-308.

Segall, S. D., Artz, W. E., Raslan, D. S., Ferraz, V. P., \& Takahashi, J. A. (2006). Triacylglycerol analysis of pequi (Caryocar brasiliensis Camb.) oil by electrospray and tandem mass spectrometry. Journal of the Science of Food and Agriculture, 86(3), 445-452.

Singh, R., Kaur, N., Kishore, L., \& Gupta, G. K. (2013). Management of diabetic complications: a chemical constituents based approach. Journal of ethnopharmacology, 150(1), 51-70.

Singh, V. P., Bali, A., Singh, N., \& Jaggi, A. S. (2014). Advanced glycation end products and diabetic complications. The Korean journal of physiology \& pharmacology, 18(1), 1-14.

Sociedade Brasileira de Diabetes. (2015). Diretrizes da sociedade brasileira de diabetes. Diretrizes SBD.

Sousa, T. M. M. (2012). Efeito antioxidante do óleo de pequi em cápsula nos pacientes com lúpus eritematoso sistêmico. Dissertação (Mestrado - Programa de Pós-Graduação em Ciências Médicas da Faculdade de Medicina). Universidade de Brasília, Brasília.

Souza, C. F. D., Gross, J. L., Gerchman, F., \& Leitão, C. B. (2012). Pré-diabetes: diagnóstico, avaliação de complicações crônicas e tratamento. Arquivos Brasileiros de Endocrinologia \& Metabologia, 56, 275-284.

Townsend, D. M., Tew, K. D., \& Tapiero, H. (2003). The importance of glutathione in human disease. Biomedicine \& pharmacotherapy, 57(3-4), 145-155.

Vellosa, J. C. R., Parabocz, G. C., Manente, F. A., Ribas, J. T., \& Lima, L. W. (2013). Alterações metabólicas e inflamatórias em condições de estresse oxidativo. Revista de Ciências Farmacêuticas Básica e Aplicada, 34(3).

Vera, R., Naves, R. V., do Nascimento, J. L., Chaves, L. J., Leandro, W. M., \& de Souza, E. R. B. (2005). Caracterização física de frutos do pequizeiro (Caryocar brasiliense Camb.) no Estado de Goiás. Pesquisa Agropecuária Tropical, 35(2), 71-79.

Vera, R., de Souza, E. R. B., Fernandes, E. P., Naves, R. V., Júnior, M. S. S., Caliari, M., \& Ximenes, P. A. (2007). Caracterização física e química de frutos do pequizeiro (Caryocar brasiliense Camb.) oriundos de duas regiões no estado de Goiás, Brasil. Pesquisa Agropecuária Tropical, 37(2), 93-99.

Vieira, R. F., Agostini-Costa, T. D. S., Silva, D. D., Sano, S. M., \& Ferreira, F. R. (2010). Frutas nativas da região Centro-Oeste do Brasil. Embrapa Informação Tecnológica.

Vilas Boas, E. V. B. (2004). Frutos minimamente processados: pequi. Anais Encontro Nacional Sobre Processamento Mínimo De Frutas E Hortaliças, 3, 122127.

Volp, A. C. P., Alfenas, R. D. C. G., Costa, N. M. B., Minim, V. P. R., Stringueta, P. C., \& Bressan, J. (2008). Capacidade dos biomarcadores inflamatórios em predizer a síndrome metabólica: Inflammation biomarkers capacity in predicting the metabolic syndrome. Arquivos Brasileiros de Endocrinologia \& Metabologia, 52, 537-549. 
Research, Society and Development, v. 10, n. 11, e321101119603, 2021

(CC BY 4.0) | ISSN 2525-3409 | DOI: http://dx.doi.org/10.33448/rsd-v10i11.19603

Vosgerau, D. S. A. R. \& Romanowski, J. P. (2014) Estudos de revisão: implicações conceituais e metodológicas. Revista de Diálogo Educacional, (14)41, 165-189.

Wedick, N. M., Pan, A., Cassidy, A., Rimm, E. B., Sampson, L., Rosner, B., \& van Dam, R. M. (2012). Dietary flavonoid intakes and risk of type 2 diabetes in US men and women. The American journal of clinical nutrition, 95(4), 925-933.

Winkelmann, E. R., \& Fontela, P. C. (2014). Condições de saúde de pacientes com diabetes mellitus tipo 2 cadastrados na Estratégia Saúde da Família, em Ijuí, Rio Grande do Sul, 2010-2013. Epidemiologia e Serviços de Saúde, 23, 665-674.

Wu, W., Wang, M., Sun, Z., Wang, X., Miao, J., \& Zheng, Z. (2012). The predictive value of TNF- $\alpha$ and IL-6 and the incidence of macrovascular complications in patients with type 2 diabetes. Acta diabetologica, 49(1), 3-7.

Yamagishi, S. I. (2011). Papel dos produtos de glicação avançada (AGEs) e receptor de AGEs (RAGE) em danos vasculares em diabetes. Experimental Gerontology, 46(1), 217-224. 